\title{
On Synchronization of Quasiperiodic Oscillations
}

\author{
A. D. Morozov，K. E. Morozov
}

We study the role of quasi-periodic perturbations in systems close to two-dimensional Hamiltonian ones. Similarly to the problem of the influence of periodic perturbations on a limit cycle, we consider the problem of the passage of an invariant torus through a resonance zone. The conditions for synchronization of quasi-periodic oscillations are established. We illustrate our results using the Duffing - van der Pol equation as an example.

Keywords: resonances, quasi-periodic, periodic, synchronization, averaged system, phase curves, equilibrium states

\section{Problem statement}

In 1930 A. Andronov and A. Witt investigated the problem of oscillation synchronization using the quasi-linear van der Pol equation as an example [1]. In other words, they found what is happening to a limit cycle when the system undergoes a periodic (harmonic) perturbation. Then a more general problem of synchronization in systems close to two-dimensional nonlinear Hamiltonian ones was considered [2,3]. It was established that, in contrast to the quasi-linear case, when the synchronization interval with respect to a parameter is small along with the perturbation, in the nonlinear case it is of the order $O(1)$. Recently, physicists found the effect of synchronization of quasi-periodic oscillations in the case of more sophisticated self-oscillating systems (see, for example, [4]).

Received May 11, 2018

Accepted June 22, 2018

This work was supported by the Russian Foundation for Basic Research under grants nos. 18-01-00306, 16-01-00364 and by the Russian Science Foundation under grant no. 14-41-00044, by the Ministry of Education and Science of the Russian Federation (project no. 1.3287.2017/PCh).

\author{
Albert D. Morozov \\ morozov@mm. unn.ru \\ Kirill E. Morozov \\ kirwamath@gmail.com \\ Lobachevsky State University of Nizhny Novgorod \\ prosp. Gagarina 23, Nizhny Novgorod, 603950, Russia
}


In the present paper we shall focus on the problem of synchronization of quasi-periodic oscillations in nonlinear systems close to Hamiltonian ones. We shall follow the paper [5]. So, let us consider the system

$$
\begin{aligned}
& \dot{x}=\frac{\partial H(x, y)}{\partial y}+\varepsilon g(x, y, t), \\
& \dot{y}=-\frac{\partial H(x, y)}{\partial x}+\varepsilon f(x, y, t),
\end{aligned}
$$

where $\varepsilon$ is a small positive parameter, $H$ is the Hamiltonian function and $g, f$ are sufficiently smooth (analytic) and uniformly bounded in some domain $G \subset R^{2}$ or $G \subset R^{1} \times S^{1}$ along with partial derivatives up to the second order. Besides, functions $g, f$ are continuous and quasi-periodic (as functions of time) uniformly with respect to $(x, y) \in G$ with incommensurable frequencies $\omega_{1}, \omega_{2}, \ldots, \omega_{m}$.

Let us assume that the unperturbed system is nonlinear and has a cell $D \subset G$ filled with closed phase curves $H(x, y)=h, h \in\left[h_{-}, h_{+}\right]$. We also suppose that $D$ does not contain any equilibria and separatrices. So $D$ is a connected domain homeomorphic to a ring.

In $D$ we change the variables from $x, y$ to the action $I$-angle $\theta$ variables. Then the system (1.1) takes the form

$$
\begin{aligned}
\dot{I} & =\varepsilon F_{1}\left(I, \theta, \theta_{1}, \ldots, \theta_{m}\right), \\
\dot{\theta} & =\omega(I)+\varepsilon F_{2}\left(I, \theta, \theta_{1}, \ldots, \theta_{m}\right), \\
\dot{\theta_{k}} & =\omega_{k}, \quad k=1,2, \ldots, m,
\end{aligned}
$$

where

$$
\begin{aligned}
& F_{1}=f\left(x(I, \theta), y(I, \theta), \theta_{1}, \ldots, \theta_{n}\right) x_{\theta}^{\prime}-g\left(x(I, \theta), y(I, \theta), \theta_{1}, \ldots, \theta_{n}\right) y_{\theta}^{\prime}, \\
& F_{2}=-f\left(x(I, \theta), y(I, \theta), \theta_{1}, \ldots, \theta_{n}\right) x_{I}^{\prime}+g\left(x(I, \theta), y(I, \theta), \theta_{1}, \ldots, \theta_{n}\right) y_{I}^{\prime},
\end{aligned}
$$

the functions $x(I, \theta), y(I, \theta)$ define the change of variables.

System (1.2) is defined on the direct product of the interval $K \times T^{m+1}$, where $K=$ $=\left[I_{-}\left(h_{-}\right), I_{+}\left(h_{+}\right)\right]$and $T^{m+1}$ is an $(m+1)$-dimensional torus, where $h_{-}, h_{+}$determine the boundaries of $D$. Here $\omega(I)$ is a natural frequency of the unperturbed system. We shall suppose $\omega(I)$ to be monotonic over the interval $\left[I_{-}, I_{+}\right]$.

Along with the system (1.1), let us consider the autonomous system

$$
\begin{aligned}
& \dot{x}=\frac{\partial H}{\partial y}+\varepsilon \bar{g}(x, y) \\
& \dot{y}=-\frac{\partial H}{\partial x}+\varepsilon \bar{f}(x, y),
\end{aligned}
$$

where

$$
\begin{aligned}
& \bar{g}(x, y)=\frac{1}{(2 \pi)^{m}} \int_{0}^{2 \pi} \ldots \int_{0}^{2 \pi} g\left(x, y, \theta_{1}, \ldots, \theta_{m}\right) d \theta_{1} \ldots d \theta_{m}, \\
& \bar{f}(x, y)=\frac{1}{(2 \pi)^{m}} \int_{0}^{2 \pi} \ldots \int_{0}^{2 \pi} f\left(x, y, \theta_{1}, \ldots, \theta_{m}\right) d \theta_{1} \ldots d \theta_{m} .
\end{aligned}
$$

Suppose that this system has a rough limit cycle. It implies that the generating PoincaréPontryagin function $B(h)$ has a simple zero $h=h_{0}[3]$. 
In [5] the solutions behavior in neighborhoods of individual levels of energy (both resonance and nonresonance ones) was established. Here we shall study the solutions' behavior in a neighborhood of the level $h=h_{0}$ (such that $B\left(h_{0}\right)=0$ ) when it is close to the resonance one. Actually, we consider the synchronization problem in the case of quasi-periodic perturbations.

As an example we take the following system:

$$
\begin{aligned}
& \dot{x}=y, \\
& \dot{y}=-x-x^{3}+\varepsilon\left[\left(1-p_{1} x^{2}\right) y+p_{2} F(t)\right],
\end{aligned}
$$

which is equivalent to the Duffing - van der Pol equation. Here $p_{1}>0, p_{2}$ are parameters, $F(t)=\sin \omega_{1} t \sin \omega_{2} t, \omega_{1}=1, \omega_{2}=\sqrt{5}$.

\section{Behavior of solutions in neighborhoods of resonance levels}

For $\varepsilon=0$, the $(m+2)$-dimensional phase space of system $(1.2)$ is foliated by $(m+1)$-dimensional tori $T_{m+1}$, the motion on which is conditionally periodic with frequencies $\omega_{1}, \ldots, \omega_{m}$. For $\varepsilon \neq 0$, the invariant tori are destroyed owing to the nonconservativity of the perturbation and/or the presence of an integer combination of the frequencies $\omega, \omega_{1}, \ldots, \omega_{m}$ :

$$
n \omega(I)-(\mathbf{k}, \Omega)=0, \quad \mathbf{k}=\left(k_{1}, \ldots, k_{m}\right), \quad \Omega=\left(\omega_{1}, \ldots, \omega_{m}\right) .
$$

For given $\Omega$ and fixed $\mathbf{k}, n$, relation (2.1) can be viewed as an equation for $I$. If this equation has a real solution $I=I_{n k}$ on the interval $\left[I_{-}, I_{+}\right]$, then the level $I=I_{n k}$ (the closed phase curve $H(x, y)=h_{n k}$ of the unperturbed system) will be called a resonance level.

Let us proceed to the study of the behavior of solutions in the neighborhoods $U_{\mu}=$ $=\left\{(I, \theta): I_{n \mathbf{k}}-C \mu<I<I_{n \mathbf{k}}+C \mu, 0 \leqslant \theta<2 \pi, C=\right.$ const $\left.>0, \mu=\sqrt{\varepsilon}\right\}$ of the individual resonance levels $I=I_{n k}$. In system (1.2), we make the change of variables

$$
\theta=\psi+\sum_{j=1}^{m} k_{j} \theta_{j} / n, \quad I=I_{n \mathbf{k}}+\mu W, \quad \mu=\sqrt{\varepsilon} .
$$

As a result, system (1.2) becomes

$$
\begin{aligned}
\dot{W} & =\mu F_{1}\left(I_{n \mathbf{k}}, \psi+\left(\sum_{j=1}^{m} k_{j} \theta_{j}\right) / n, \theta_{1}, \ldots, \theta_{m}\right)+ \\
& +\mu^{2}\left[\partial F_{1}\left(I_{n \mathbf{k}}, \psi+\left(\sum_{j=1}^{m} k_{j} \theta_{j}\right) / n, \theta_{1}, \ldots, \theta_{m}\right) / \partial I\right] W+O\left(\mu^{3}\right), \\
\dot{\psi} & =\mu b_{1} W+\mu^{2} F_{2}\left(I_{n \mathbf{k}}, \psi+\left(\sum_{j=1}^{m} k_{j} \theta_{j}\right) / n, \theta_{1}, \ldots, \theta_{m}\right)+O\left(\mu^{3}\right), \\
\dot{\theta_{j}} & =\omega_{j}, \quad j=1, \ldots, m,
\end{aligned}
$$

where the functions $F_{1}, F_{2}, \partial F_{1} / \partial I$ are $2 \pi n$-periodic in $\theta_{1}, \theta_{2}, \ldots, \theta_{m}$. Note that since the function $\omega(I)$ is monotone, one has $b_{1}=\omega^{\prime}\left(I_{n \mathbf{k}}\right) \neq 0$ (the nondegeneracy condition for the resonance). The variables in system (2.3) are divided into the slow variables $W, \psi$ and the fast variables $\theta_{1}, \ldots, \theta_{m}$. One can represent this system in the standard form of the averaging method 
and apply the Bogolyubov theorem ([6, p. 379]; see also [7, p. 210]). Indeed, by substituting $\theta_{j}=\omega_{j} t$ into the first two equations in system (2.3), we obtain

$$
\dot{\eta}=\mu F\left(\eta, \omega_{1} t, \ldots, \omega_{m} t ; \mu\right)
$$

where $\eta=(W, \psi), F=\left(F_{1}+\mu\left(\partial F_{1} \partial I\right) W+O\left(\mu^{2}\right), b_{1} W+\mu F_{2}+O\left(\mu^{2}\right)\right)$. Then the averaged system acquires the form $\dot{\xi}=\mu F_{0}(\xi ; \mu)$, where $\xi=(u, v), u=W+O(\mu), v=\psi+O(\mu)$,

$$
F_{0}=\lim _{T \rightarrow \infty} \frac{1}{T} \int_{0}^{T} F\left(\xi, \omega_{1} t, \ldots, \omega_{m} t ; \mu\right) d t=\frac{1}{(2 \pi n)^{m}} \int_{0}^{2 \pi n} \ldots \int_{0}^{2 \pi n} F\left(\xi, \theta_{1}, \ldots, \theta_{m} ; \mu\right) d \theta_{1} \ldots d \theta_{m}
$$

owing to the incommensurability of the frequencies.

The two-dimensional averaged system becomes

$$
\begin{aligned}
& u^{\prime}=A\left(v, I_{n \mathbf{k}}\right)+\mu P_{0}\left(v, I_{n \mathbf{k}}\right) u, \\
& v^{\prime}=b_{1} u+\mu\left(b_{2} u^{2}+Q_{0}\left(v, I_{n \mathbf{k}}\right)\right),
\end{aligned}
$$

where the prime stands for the derivative with respect to the slow time $\tau=\mu t$ and

$$
\begin{aligned}
A & =\frac{1}{(2 \pi n)^{m}} \int_{0}^{2 \pi n} \ldots \int_{0}^{2 \pi n} F_{1}\left(I_{n \mathbf{k}}, v+\left(\sum_{j=1}^{m} k_{j} \theta_{j}\right) / n, \theta_{1}, \ldots, \theta_{m}\right) d \theta_{1} \ldots d \theta_{m}, \\
P_{0} & =\frac{1}{(2 \pi n)^{m}} \int_{0}^{2 \pi n} \ldots \int_{0}^{2 \pi n}\left[\partial F_{1}\left(I_{n \mathbf{k}}, v+\left(\sum_{j=1}^{m} k_{j} \theta_{j}\right) / n, \theta_{1}, \ldots, \theta_{m}\right) \partial I\right] d \theta_{1} \ldots d \theta_{m}, \\
Q_{0} & =\frac{1}{(2 \pi n)^{m}} \int_{0}^{2 \pi n} \ldots \int_{0}^{2 \pi n} F_{2}\left(I_{n \mathbf{k}}, v+\left(\sum_{j=1}^{m} k_{j} \theta_{j}\right) / n, \theta_{1}, \ldots, \theta_{m}\right) d \theta_{1} \ldots d \theta_{m}, \\
b_{1} & =d \omega\left(I_{n k}\right) / d I, \quad b_{2}=\left(d^{2} \omega\left(I_{n k}\right) / d I^{2}\right) / 2 .
\end{aligned}
$$

By analogy with the case of periodic perturbations, system (2.4) can be reduced modulo $O\left(\mu^{2}\right)$ terms to the form (see $[3,9]$ )

$$
\begin{aligned}
& u^{\prime}=A\left(v, I_{n \mathbf{k}}\right)+\mu \sigma u, \\
& v^{\prime}=b_{1} u+\mu b_{2} u^{2},
\end{aligned}
$$

where

$$
\sigma=P_{0}+\frac{d Q_{0}}{d v}=\frac{1}{(2 \pi n)^{m}} \int_{0}^{2 \pi n} \ldots \int_{0}^{2 \pi n}\left(g_{x}^{\prime}+f_{y}^{\prime}\right) d \theta_{1} \ldots d \theta_{m} .
$$

Here $x=x\left(I_{n \mathbf{k}}, v+\left(\sum_{j=1}^{m} k_{j} \theta_{j}\right) / n\right), y=y\left(I_{n \mathbf{k}}, v+\left(\sum_{j=1}^{m} k_{j} \theta_{j}\right) / n\right)$. The functions $A\left(v, I_{n \mathbf{k}}\right)$ and $\sigma\left(v, I_{n \mathbf{k}}\right)$ are sufficiently smooth and periodic with least period $2 \pi / n$ [8, p. 158]. The phase space of system $(2.6)$ is the cylinder $\{u, v \bmod (2 \pi / n)\}$.

Associated with a simple stable equilibrium of the averaged system (2.6) is a stable quasiperiodic resonance solution with frequencies $k_{1} \omega_{1} / n, \ldots, k_{m} \omega_{m} / n[6]$. 
Let us represent $A\left(v, I_{n \mathbf{k}}\right)$ in the form ${ }^{1}$

$$
A\left(v, I_{n \mathbf{k}}\right)=\widetilde{A}\left(v, I_{n \mathbf{k}}\right)+B\left(I_{n \mathbf{k}}\right),
$$

where $B\left(I_{n \mathbf{k}}\right)=\frac{n}{2 \pi} \int_{0}^{2 \pi / n} A\left(v, I_{n \mathbf{k}}\right) d v$ is the mean of $A\left(v, I_{n \mathbf{k}}\right)$. Suppose that the level $I=I_{n \mathbf{k}}$ is an impassable one and it is close to the level $I=I_{0}$, in the neighborhood of which the system (1.4) has the rough limit cycle.

\section{Passage of the $(m+1)$-dimensional torus through the resonance zone}

Let us introduce in (2.6) the "detuning" $\gamma$ which measures the deviation of the resonance level $I=I_{n \mathbf{k}}$ from the level $I_{0}$. Following [8] and [9], we observe the transition from the exact resonance to the nonresonance case, as we change $\gamma$. In order to do that, we consider the system on a cylinder

$$
\begin{aligned}
& \frac{d u}{d \tau}=\widetilde{A}\left(v, I_{n \mathbf{k}}\right)+\mu\left(\sigma\left(v, I_{n \mathbf{k}}\right) u+\gamma\right), \\
& \frac{d v}{d \tau}=b u+\mu b_{1} u^{2},
\end{aligned}
$$

obtained from (2.6) by letting

$$
B\left(I_{n \mathbf{k}}\right)=\left(d B_{0}\left(I_{0}\right) / d I\right)\left(I_{n \mathbf{k}}-I_{0}\right)=\mu \gamma .
$$

Along with (2.6) consider the conservative system

$$
\begin{aligned}
& \frac{d u}{d \tau}=\widetilde{A}\left(v, I_{n \mathbf{k}}\right) \\
& \frac{d v}{d \tau}=b u .
\end{aligned}
$$

We call the separatrices of the saddles of (3.1) embracing the phase cylinder $(v \bmod 2 \pi, u)$ external separatrices.

Let us describe the relative positions of the external separatrices when the terms $O(\mu)$ are taken into account. We make use of the formula $\Delta_{\mu}=\mu \Delta_{1}+O\left(\mu^{2}\right)$ for the distance $\Delta_{\mu}$ between the corresponding separatrices of (2.6) [10]. Let us consider (3.2) as the unperturbed system and substitute in (3.1)

$$
v=\xi+v_{0}-\mu \gamma / \widetilde{A}^{\prime}\left(v_{0}\right), \quad u=\eta,
$$

where $v_{0}$ is the coordinate of the saddle of (3.2).

As a result, (3.1), up to the terms $O\left(\mu^{2}\right)$, assumes the form

$$
\left.\begin{array}{l}
\frac{d \eta}{d \tau}=\widetilde{A}\left(\xi+v_{0}\right)+\mu\left[\gamma+\sigma\left(\xi+v_{0}\right) \eta-\gamma \widetilde{A}^{\prime}\left(\xi+v_{0}\right) / \widetilde{A}^{\prime}\left(v_{0}\right)\right] \\
\frac{d \xi}{d \tau}=b \eta+\mu b_{1} \eta^{2}
\end{array}\right\}
$$

\footnotetext{
${ }^{1}$ An example of computation of the function $A\left(v, I_{n k}\right)$ is given below in Section 4.
}

RUSSIAN JOURNAL OF NONLINEAR DYNAMICS, 2018, 14(3), 367-376 
Evidently, the right-hand sides of (3.4) vanish when $\xi=\eta=0$. Therefore, according to [10], we have

$$
\Delta_{1}=-\int_{-\infty}^{\infty} b_{1} \eta^{2} \frac{d \eta}{d \tau} d \tau+\int_{-\infty}^{\infty}\left[\sigma\left(\xi+v_{0}\right) \eta+\gamma-\gamma \frac{\widetilde{A}^{\prime}\left(\xi+v_{0}\right)}{\widetilde{A}^{\prime}\left(v_{0}\right)}\right] \frac{d \xi}{d \tau} d \tau
$$

Here $(\xi(\tau), \eta(\tau))$ is the solution of (3.2) on the separatrix. From the integral of (3.2) we obtain the following relation between $\eta$ and $\xi$ :

$$
\eta= \pm\left[\frac{2}{b}\left(V\left(\xi, v_{0}\right)-V\left(0, v_{0}\right)\right]^{1 / 2}, \quad V\left(\xi, v_{0}\right)=\int \widetilde{A}\left(\xi+v_{0}\right) d \xi\right.
$$

Substituting (3.6) in (3.5), we find

$$
\Delta_{1}^{ \pm}=\frac{2 \pi \gamma}{n} \pm \int_{0}^{2 \pi / p} \sigma\left(\xi+v_{0}\right)\left[\frac{2}{b}\left(V\left(\xi, v_{0}\right)-V\left(0, v_{0}\right)\right)\right]^{1 / 2} d \xi
$$

According to $[8,9]$, the following prepositions hold.

Preposition 1. For the exact resonance (i.e., for $\gamma=0$ ) when the function $\sigma(v)$ has constant sign the external separatrices are split, i.e. $\Delta_{\mu} \neq 0$.

Preposition 2. When function $\sigma(v)$ has constant sign, system (3.1) with $\gamma=0$ has no limit cycles.

When $\sigma(v)$ has constant sign, from the conditions $\Delta_{1}^{ \pm}=0$ we find the (bifurcational) values of the "detuning" $\gamma=\gamma^{ \pm}$corresponding to the appearance in (3.1) of a separatrix going from a saddle to a saddle. The plus in $\gamma^{ \pm}$corresponds to the domain $\eta>0$ and the minus - to $\eta<0$. As $\gamma$ goes past $\gamma^{ \pm}$, a noncontractible limit cycle is born in (3.1).

Theorem 1. Let $\sigma\left(v, I_{n \mathbf{k}}\right)$ have constant sign, and assume, for concreteness, that $\varepsilon \sigma<0$. Then there exists $\gamma_{n \mathbf{k}}=\left|\gamma^{ \pm}\right|+O(\mu)$ such that:

1) for $\gamma>\gamma_{n \mathbf{k}} E q$. (3.1) has a noncontractible stable limit cycle;

2) for $\gamma=\gamma_{n \mathbf{k}}$ the limit cycle "sticks" to the separatrix contour $\Gamma_{n}^{+}$formed by $n$ saddles and external separatrices connecting one saddle to another, while the other unstable separatrices of the saddles approach stable focuses as $t \rightarrow \infty$;

3) for $-\gamma_{n \mathbf{k}}<\gamma<\gamma_{n \mathbf{k}}$ there are no noncontractible limit cycles;

4) for $\gamma=-\gamma_{n \mathbf{k}}$ there appears a contour $\Gamma_{n}^{-}$, formed by $n$ saddles and their external separatrices, whose direction and position differ from those of $\Gamma_{n}^{+}$;

5) for $\gamma<-\gamma_{n \mathbf{k}}$ there is a stable noncontractible limit cycle.

Figure 1 illustrates this theorem.

In fact, the theorem describes the "passage" of the $(m+1)$-dimensional torus through a resonance zone. Indeed, the limit cycle of (3.1), embracing the phase cylinder, corresponds to the $(m+1)$-dimensional torus in the initial (1.1) as well as the simple equilibrium corresponds to the $m$-dimensional torus (there is a quasi-periodic solution with the frequencies $\left.k_{1} \omega_{1} / n, k_{2} \omega_{2} / n, \ldots, k_{m} \omega_{m} / n\right)[5,6]$. 


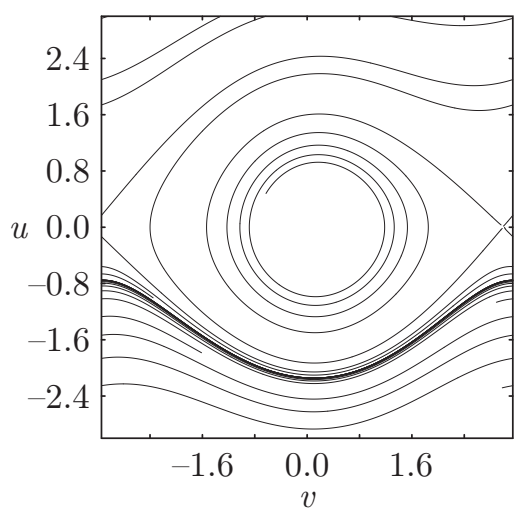

(a)

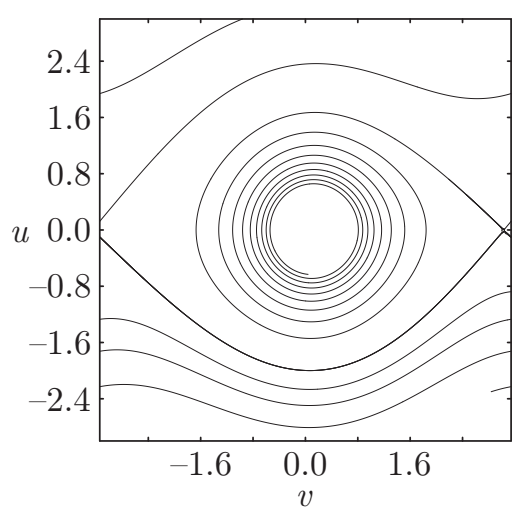

(b)

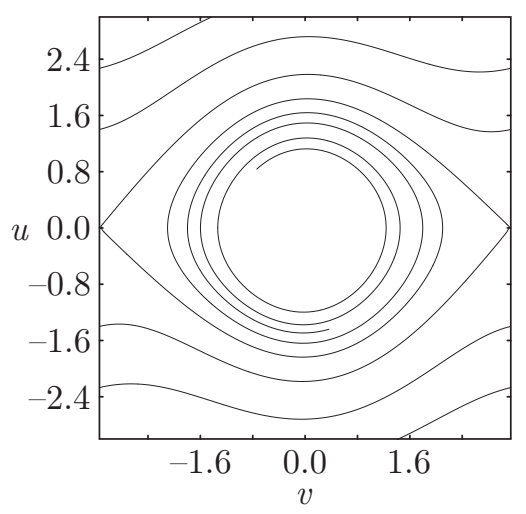

(c)

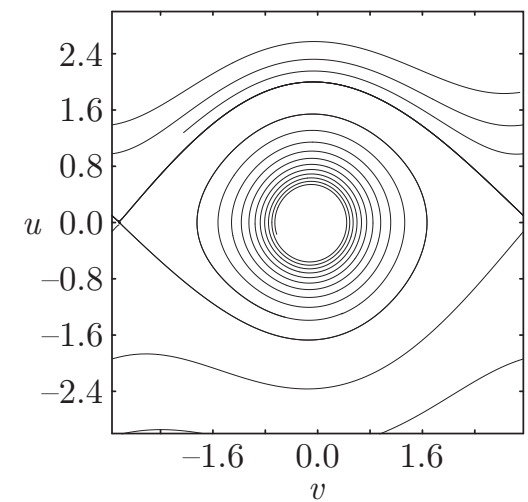

(d)

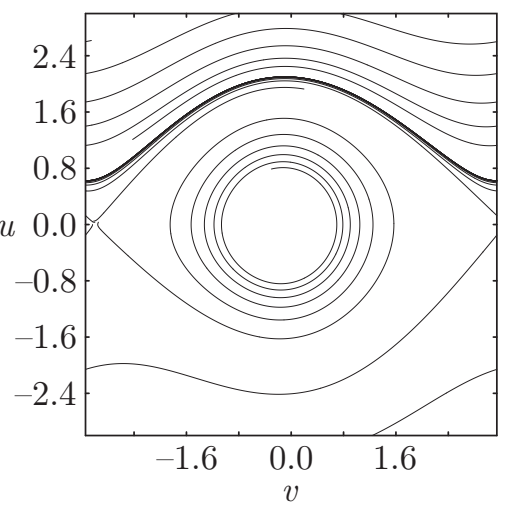

(e)

Fig. 1. Phase portraits of (3.1) under variation of $\gamma$.

\section{Example}

Let us study system (1.5). The unperturbed system $(\varepsilon=0)$ admits the following integral of energy $H(x, y)=y^{2} / 2+x^{2} / 2+x^{4}=h, h>0$. Denote the annular region of the phase plane $0<h_{-} \leqslant h \leqslant h_{+}<\infty$ by $D$.

The unperturbed system is equivalent to the second-order equation that has the following solution:

$$
x(\theta, I)=x_{1} \operatorname{cn}(2 \mathbf{K} \theta / \pi), \quad \theta=\omega t,
$$

where $\omega=\pi(1+4 h)^{1 / 4} /(2 \mathbf{K})$ is the frequency of motion on closed phase curves $H=h(I)$, $x_{1}$ is a positive solution of the equation $x^{2} / 2+x^{4} / 4=h, \operatorname{cn}(u)$ is the Jacobi elliptic function, $\mathbf{K}$ is the complete elliptic integral of first kind, and $k=k(h)$ is its modulus.

\subsection{Autonomous system}

First let us consider the autonomous system

$$
\dot{x}=y, \dot{y}=-x-x^{3}+\varepsilon\left[\left(1-p_{1} x^{2}\right) y\right] .
$$

The Poincaré - Pontryagin generating function has the form (up to a positive factor) [9]

$$
B(\rho)=\left[(1-\rho)(1-2 \rho) \mathbf{K}-(1-2 \rho)^{2} \mathbf{E}\right]+\frac{2 p_{1}}{5}\left[(\rho-1)(2-\rho) \mathbf{K}+2\left(\rho^{2}-\rho+1\right) \mathbf{E}\right],
$$


where $\mathbf{E}$ is the complete elliptic integral of the second kind, $\rho=k^{2}=\frac{-1+\sqrt{1+4 h}}{2 \sqrt{1+4 h}}$. For $p_{1}>0$ it has a unique zero (denote it by $\rho_{*} \in(0,1 / 2)$ ). As is well known [9], the simple zeros of the generating function determine the energy levels, in the neighborhood of which system (4.2) has a rough limit cycle for small enough $\varepsilon>0$. It is not hard to see that $\rho_{*} \rightarrow 0$ as $p_{1} \rightarrow \infty$ and $\rho_{*} \rightarrow 1 / 2$ as $p_{1} \rightarrow 0+$. Since $\sigma=B^{\prime}\left(\rho_{*}\right)<0$, the cycle is stable, where

$$
\sigma=1-p_{1} \frac{2}{(1-2 \rho) \mathbf{K}}[\mathbf{E}-(1-\rho) \mathbf{K}] .
$$

From the condition $B\left(\rho, p_{1}\right)=0$ we find

$$
p_{1}(\rho)=\frac{5}{2} \frac{(1-\rho)(1-2 \rho) \mathbf{K}-\left(1-2 \rho^{2}\right) \mathbf{E}}{(\rho-1)(2-\rho) \mathbf{K}+2\left(\rho^{2}-\rho+1\right) \mathbf{E}} .
$$

Figure 2 shows the fragments of the dependence $p_{1}(\rho)$ in neighborhoods of resonances with $n=1$ (a) and $n=3(\mathrm{~b})$.

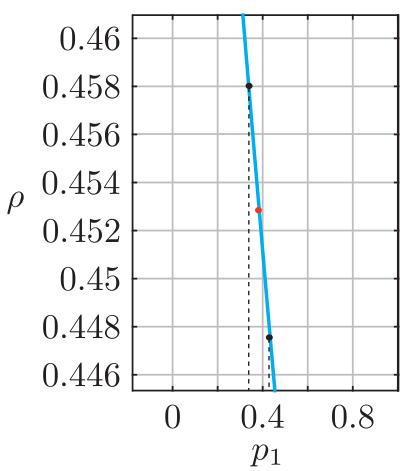

(a)

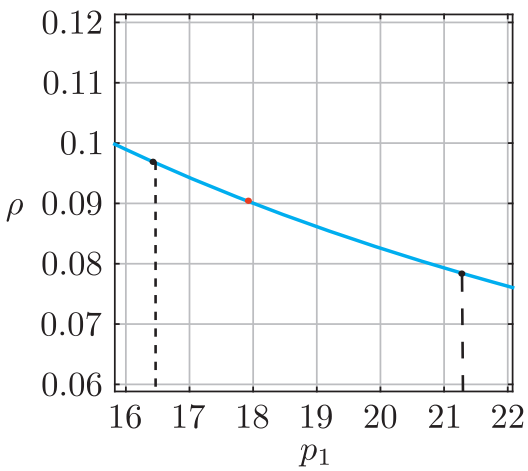

(b)

Fig. 2. Fragments of the dependence $p_{1}(\rho)$.

\subsection{Resonance zones and synchronization}

Let us consider the resonance level $I=I_{n, k_{1} k_{2}}$ determined by the condition $n \omega(h(I))=$ $=k_{1} \omega_{1}+k_{2} \omega_{2}$.

The pendulum type equation describing the topology of the resonance zone has the form

$$
\frac{d^{2} v}{d \tau^{2}}-A(v)=\mu \sigma \frac{d v}{d \tau}
$$

Just as before, we represent the function $A(v)$ in the form

$$
A\left(v ; I_{n k_{1} k_{2}}\right)=B\left(I_{n k_{1} k_{2}}\right)+p_{2} S\left(v ; I_{n k_{1} k_{2}}\right),
$$

where

$$
S\left(v ; I_{n k_{1} k_{2}}\right)=\frac{1}{4 \pi^{2} n^{2}} \int_{0}^{2 \pi n} \int_{0}^{2 \pi n} x_{\theta}^{\prime}\left(\theta, I_{n k_{1} k_{2}}\right) \sin \theta_{1} \sin \theta_{2} d \theta_{1} d \theta_{2} .
$$




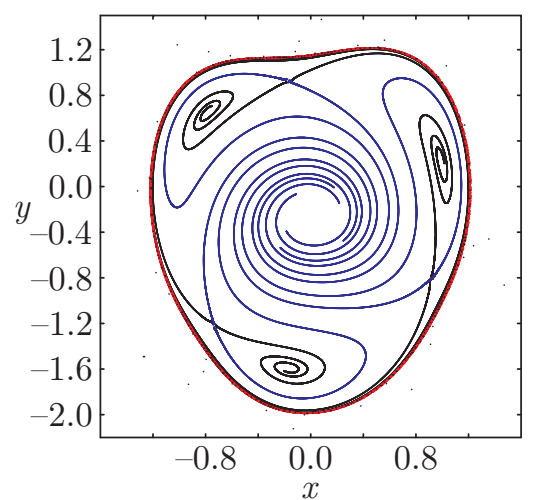

(a)

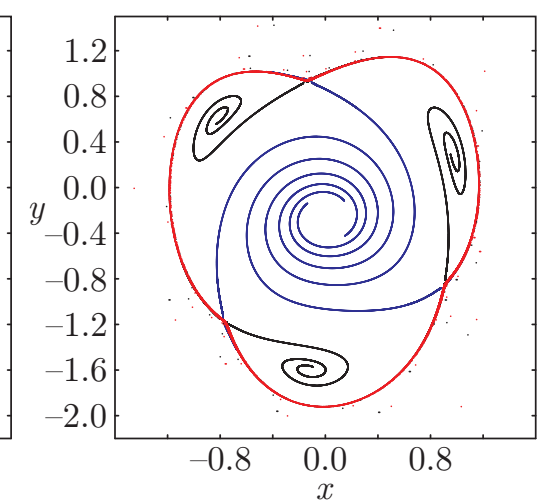

(b)

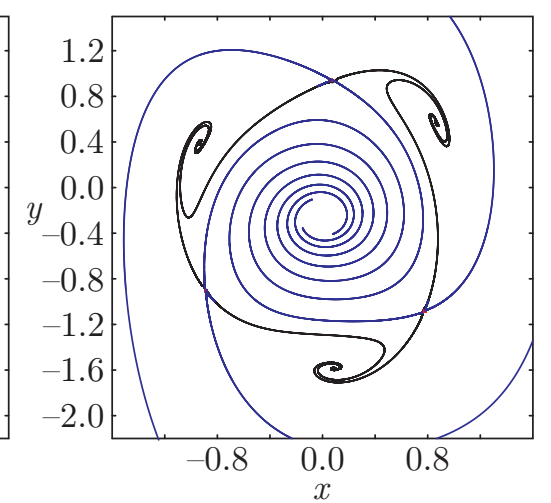

(c)

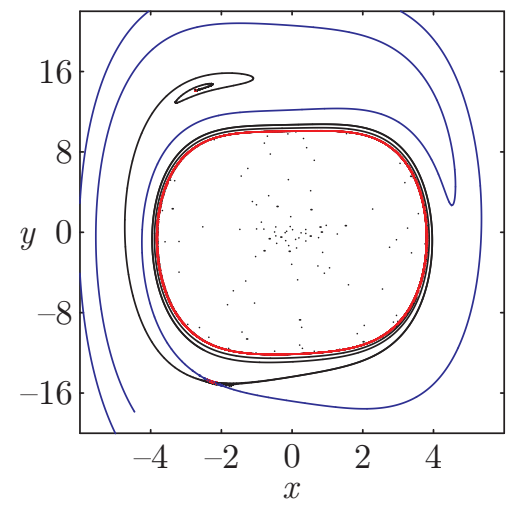

(d)

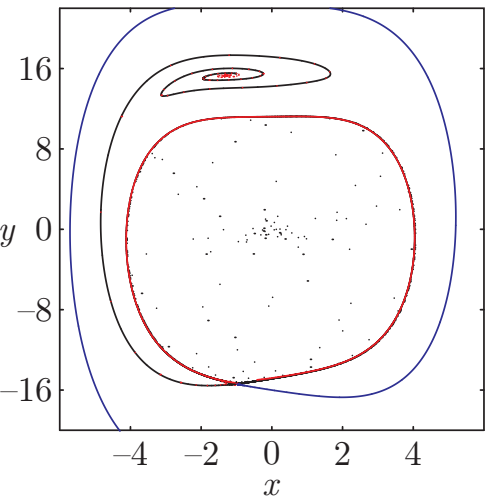

(e)

Fig. 3. Phase portraits of (4.6) in the $(x, y)$-plane in the neighborhood of the level $h_{311}$ : (a), (b), (c) and $h_{111}:(\mathrm{d}),(\mathrm{e})$.

Computing the function $S(v)$, we obtain the following expressions:

$$
A\left(v ; I_{n k_{1} k_{2}}\right)=\left\{\begin{aligned}
p_{2} \sqrt{2}\left(\omega_{1}+\omega_{2}\right) \frac{a^{n / 2}}{1+a^{n}} \sin n v+B\left(I_{n k_{1} k_{2}}\right) & \\
& \text { if } n \text { is odd, } k_{1}=k_{2}=1 \\
B\left(I_{n k_{1} k_{2}}\right) ; & \text { if } n \text { is even or } k_{1} \neq 1, k_{2} \neq 1
\end{aligned}\right.
$$

where $a=\exp \left(-\frac{\pi \mathbf{K}\left(\sqrt{1-k^{2}}\right)}{\mathbf{K}(k}\right)$.

From the conditions $\omega=(1+\sqrt{5}) / n$ and $\omega>1$ it follows that there can be only two splittable resonance levels $(n=1, n=3)$.

Figure 3 shows the phase portraits of Eq. (4.5) in the $(x ; y)$-plane under variation of $p_{1}$ in the neighborhood of resonance levels of energy.

The intervals of synchronization of quasi-periodic oscillations with respect to $p_{1}$ (see on Fig. 2) could be obtained similarly to the case of periodic perturbations [8, pp. 184-187].

Rough equilibria of (4.6) correspond to the resonance quasi-periodic solutions with frequencies $\omega_{1} / n, \omega_{2} / n, n=1, n=3$ of the initial system (1.5). Such a solution for $p_{1}=18, p_{2}=1$, $\varepsilon=0.05$ and $n=3$ is presented in Fig. 4a. The projection of this solution onto the $(x, y)$-plane is shown in Fig. 4b. 


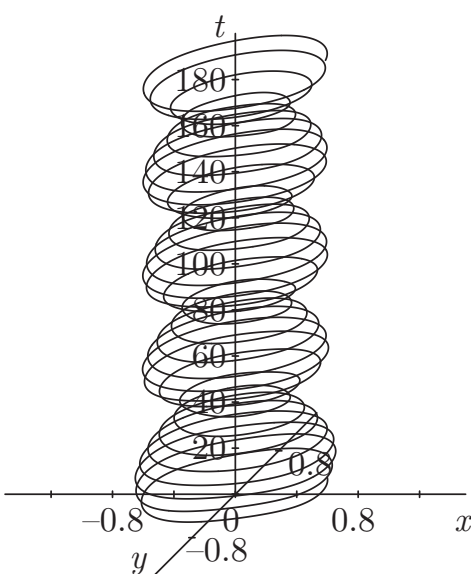

(a)

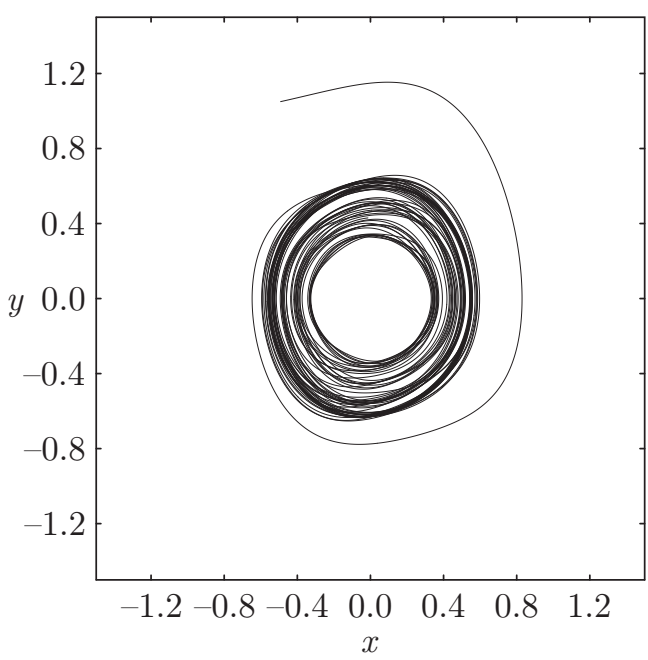

(b)

Fig. 4

\section{References}

[1] Andronov, A. A. and Witt, A.A., Zur Theorie des Mitnehmens von van der Pol, Arch. für Elektrotech., 1930, vol. 24, no. 1, pp. 99-110.

[2] Morozov, A. D. and Shil'nikov, L. P., To Mathematical Theory of Oscillatory Synchronization, Dokl. Akad. Nauk SSSR, 1975, vol. 223, no. 6, pp. 1340-1343 (Russian).

[3] Morozov, A. D. and Shil'nikov, L. P., On Nonconservative Periodic Systems Close to Two-Dimensional Hamiltonian, J. Appl. Math. Mech., 1983, vol.47, no. 3, pp. 327-334; see also: Prikl. Mat. Mekh., 1983, vol. 47, no. 3, pp. 385-394.

[4] Anishenko, V.S. and Nikolaev, S. M., Experimental Research of Synchronization of Two-Frequency Quasiperiodic Motions, Izv. Vyssh. Uchebn. Zaved. Prikl. Nelin. Dinam., 2007, vol.15, no.6, pp. 93-101 (Russian).

[5] Morozov, A. D. and Morozov, K. E., Quasiperiodic Perturbations of Two-Dimensional Hamiltonian Systems, Differ. Equ., 2017, vol.53, no.12, pp.1557-1566; see also: Differ. Uravn., 2017, vol.53, no. 12, pp. 1607-1615.

[6] Bogoliubov, N. N. and Mitropolsky, Yu. A., Asymptotic Methods in the Theory of Non-Linear Oscillations, New York: Gordon \& Breach, 1961.

[7] Mitropolsky, Yu. A. and Lykova, O. B., Integrated Manifolds in the Nonlinear Mechanics, Moscow: Nauka, 1973 (Russian).

[8] Morozov, A.D., Quasi-Conservative Systems: Cycles, Resonances and Chaos, World Sci. Ser. Nonlinear Sci. Ser. A Monogr. Treatises, vol.30, River Edge, N.J.: World Sci., 1999.

[9] Morozov, A. D., Resonance, Cycles and Chaos in Quasi-Conservative Systems, Izhevsk: R\&C Dynamics, Institute of Computer Science, 2005 (Russian).

[10] Mel'nikov, V. K., On the Stability of a Center for Time-Periodic Perturbations, Tr. Mosk. Mat. Obs., 1963, vol. 12, pp. 3-52 (Russian). 